\title{
ENHANCEMENT OF FRACTURE HEALING AFTER CITRULLINE SUPPLEMENTATION IN MICE
}

\author{
D.M. Meesters ${ }^{1,2, *}$, P.F.W. Hannemann¹, H.M.H. van Eijk ${ }^{1,2}$, V.T.J. Schriebl ${ }^{1}$, P.R.G. Brink ${ }^{1}$, M. Poeze ${ }^{1,2}$ \\ and K.A.P. Wijnands ${ }^{1,2}$ \\ ${ }^{1}$ Department of General Surgery and Trauma Surgery, Maastricht University Medical Centre, \\ Maastricht, the Netherlands \\ ${ }^{2}$ NUTRIM School for Nutrition and Translational Research In Metabolism, Maastricht University, \\ Maastricht, the Netherlands
}

\begin{abstract}
Around $10 \%$ of long bone fractures show inadequate bone healing resulting in non-union development. A deregulated arginine-citrulline-nitric oxide metabolism caused by a poor nutritional status of the patients is a risk factor for non-unions. Additionally, previous research in mice with a disrupted arginine to citrulline conversion showed delayed healing. The study hypothesis was that stimulating said metabolism could positively influence the healing process through promotion of collagen synthesis and angiogenesis.

Adult wild-type mice underwent a femur osteotomy and plate-screw osteosynthesis. Mice were randomly divided into three groups and received daily oral supplementation of arginine, citrulline or $0.9 \%$ saline (control). Body weight and food intake were measured daily. After $14 \mathrm{~d}$, the mice were euthanised and femora collected. Callus formation was assessed by micro-computed tomography and concentrations of amino acids and enzymes in the femora were measured.

Only citrulline-treated mice showed significantly increased bridging of the fracture gap when compared to control mice. Femur citrulline and ornithine concentrations were increased in citrulline-treated animals. qPCR showed significantly decreased expression of inflammatory markers, whereas increased expression of angiogenic and collagen-producing factors was observed in citrulline-treated mice. Although food intake did not show any difference between the three groups, animals treated with citrulline showed a weight gain of $0.3 \mathrm{~g}$, compared with a $0.1 \mathrm{~g}$ decline in the control group.

Daily oral citrulline supplementation stimulated callus formation and improved the inflammatory response, positively contributing to the enhanced healing response. Finally, the increased weight gain pointed toward a better post-operative recovery.
\end{abstract}

Keywords: Non-union, citrulline, arginine, nitric oxide, fracture healing.

*Address for correspondence: Dennis M. Meesters, Maastricht University Medical Centre, Department of Surgery and Trauma Surgery, PO Box 5800, 6202 AZ Maastricht, the Netherlands.

Telephone number: +31 433881891 Email: d.meesters@maastrichtuniversity.nl

Copyright policy: This article is distributed in accordance with Creative Commons Attribution Licence (http://creativecommons.org/licenses/by-sa/4.0/).

\section{Introduction}

Every year, about $1 \%$ of the population sustains a fracture (Mills et al., 2017; van Staa et al., 2001). Generally, fractures heal without any complication, however, in around $10 \%$ of all long-bone fractures, difficulties occur during the healing process resulting in delayed union or non-union formation (Bastian et al., 2016; Court-Brown and McQueen, 2008). Next to the fracture location, the degree of (soft) tissue injury, the type and quality of (surgical) treatment as well as several patient-dependent risk factors are known to contribute to non-union development.
Besides the use of certain drugs [e.g. nonsteroidal anti-inflammatory drugs (NSAIDs)] and a disturbed vascularity (smoking, diabetes), malnutrition is one of the main risk factors for an impaired fracture healing process (Bishop et al., 2012; Calori et al., 2007).

One of the main metabolic processes influencing bone healing is the arginine-citrulline-nitric oxide (NO)-metabolism (Meesters et al., 2018). During physiological conditions, the semi-essential amino acid arginine is produced by conversion of citrulline by the cytosolic enzymes arginine succinate lyase and arginine succinate synthetase. Arginine can be converted back into citrulline by one of the 
nitric oxide synthase (NOS) enzymes. During the inflammatory phase of fracture healing, the inducible NOS (Nos2) is active and mainly localised in the intramembranous region along the periosteal callus. During the later phases of the healing process, the constitutive and calcium-dependent endothelial NOS (Nos3) and neuronal NOS (Nos1) are expressed in the blood vessel lining and fibrous and cartilaginous tissues (Diwan et al., 2000; Zhu et al., 2001; Zhu et al., 2002). During these conversions the free radical $\mathrm{NO}$ is formed. NO stimulates bone cells to regulate bone remodelling by influencing osteoblasts as well as osteoclasts and, on the other hand, influences vascular reactivity (Chae et al., 1997; Corbett et al., 1999).

The second process within the arginine-citrullineNO-metabolism that plays an important role during fracture healing is the conversion of arginine into ornithine by the arginase- 1 enzyme. Through the formation of polyamines, ornithine is a precursor for collagen synthesis, necessary for osteogenesis (Vittur et al., 1986). A schematic representation overviewing the arginine-citrulline-NO metabolism in shown in Fig. 1.

In addition to the previously shown effect, disturbances in the arginine substrate metabolism results in non-union formation (Meesters et al., 2016). The study hypothesis was that stimulation of the arginine-citrulline-NO metabolism by oral supplementation of arginine or citrulline, as a precursor for arginine, would positively influence the fracture healing process in mice.

\section{Materials and Methods}

Animals and surgical procedure

Skeletally mature 20 to 24-week old female C57BL6/J wild type mice were used. Before starting the experiments, all animals were allowed to acclimatise for at least 2 weeks during which they were housed (4-5 animals) in individually ventilated cages on a $12 \mathrm{~h}$ light-dark cycle. During the entire experimental period, mice were fed water and standard chow ad libitum. The experimental protocols and surgical procedures were evaluated and approved by the local animal experimental ethics and welfare committee (permit number DEC 2014-030).

Before surgery, anaesthesia was induced by placing the animals in a box flooded with isoflurane. Intraoperative analgesia was administered by subcutaneous injection of $0.1 \mathrm{mg} / \mathrm{kg}$ buprenorphine. Mice were kept on a heating pad connected to a rectal probe to maintain adequate body temperature and prevent hypothermia during the surgical procedure. Inhalation anaesthesia during surgery was maintained using 1.5-2 \% isoflurane. Mice were placed in a prone position in an aseptic surgical field. The procedure was started by performing a lateral skin incision on the left femur, from the tail going towards the knee. By blunt dissection between the biceps and quadriceps femoris muscles, the femur was exposed. A $7 \times 1.5 \times 0.5 \mathrm{~mm}$ plate was placed on the femur and fixed using four $2.0 \mathrm{~mm}$ angular stable screws after predrilling using a $0.33 \mathrm{~mm}$ drill bit. After fixation, a $0.45 \mathrm{~mm}$ osteotomy was performed using a Gigli wire saw and irrigation with $0.9 \% \mathrm{NaCl}$. All screws were untightened by half a turn to induce secondary fracture healing. The MouseFix ${ }^{\mathrm{TM}}$ screws, Gigli saw, plates and instruments were obtained from RISystems, Davos, Switzerland. Skin and fascia were closed using a 5-0 vicryl (Ethicon).

The surgical procedure has previously been described by Grongroft et al. (2019). In the first $2 \mathrm{~d}$ after surgery, mice received $0.1 \mathrm{mg} / \mathrm{kg}$ subcutaneous buprenorphine every $10-14 \mathrm{~h}$ as post-operative analgesia. Animal well-being was monitored daily along with body weight registration. Mice were

\begin{tabular}{|cc|}
\hline VASCULAR REACTIVITY & BONE \& \\
$\&$ & CARTILAGE \\
INFLAMMATION & METABOLISM \\
\hline
\end{tabular}



Fig. 1. Arginine-citrulline-NO metabolism. Schematic overview of the arginine-citrulline-NO metabolism with key amino acids and enzymes and its relation with inflammation and vascular reactivity versus bone and cartilage metabolism. 
housed solitarily in individually ventilated cages for the purpose of adequate food intake measurements.

Daily supplementation of citrulline or arginine was performed by oral gavage. Citrulline was given at a concentration of $5 \mathrm{~g} / \mathrm{kg}$ bodyweight per day. The amount of arginine was $4.6 \mathrm{~g} / \mathrm{kg}$ bodyweight per day, a concentration isonitrogenous when compared with citrulline. These concentrations were extrapolated from previous studies (Poeze et al., 2011; Wijnands et al., 2021b). The volume used for oral gavage was according to the general guidelines of $10 \mathrm{~mL} /$ $\mathrm{kg}$ body weight. Although citrulline has a slightly higher caloric value when compared to arginine, this difference was only $0.02 \%$ of the average general caloric intake of mice per day. Both amino acids were dissolved in $0.9 \% \mathrm{NaCl}$. Animals in the control group only received $0.9 \% \mathrm{NaCl}$.

All mice were randomly assigned to one of the three study groups. Each group consisted of 15 animals, of which 6 were used for micro-computed tomography $(\mu \mathrm{CT})$ measurement, 6 for analysis of amino acid and enzyme concentrations and 3 for histological assessment.

After $14 \mathrm{~d}$, mice were euthanised under isoflurane anaesthesia inhalation using a cardiac puncture to collect arterial blood samples. Blood was collected on ice in pre-chilled heparinised cups (Sarstedt) and centrifuged immediately $\left(4^{\circ} \mathrm{C}, 15 \mathrm{~min}, 8,500 \times g\right)$ to obtain plasma that was, subsequently, stored at $-80^{\circ} \mathrm{C}$ for further analysis. Femora were collected for $\mu \mathrm{CT}$ by dislocation of the hip joint and storage at $4{ }^{\circ} \mathrm{C}$ until analysis. Femora which were used for RNA and amino acid measurements were cleaned from all surrounding soft tissue and snap-frozen in liquid nitrogen before storage at $-80^{\circ} \mathrm{C}$. In Fig. 2, an overview of the experimental setup is presented.

\section{Amino acid measurements}

To determine amino acid concentrations in femora, the complete frozen femora were crushed in liquid nitrogen using a pestle and mortar. Subsequently, $30 \mathrm{mg}$ of tissue were placed in $2 \mathrm{~mL}$ screw-cap vials with $0.1 \mathrm{~g}$ of glass beads ( $1 \mathrm{~mm}$ diameter) and $250 \mu \mathrm{L}$ of $5 \%$ ice-cold sulphosalicylic acid solution for deproteinisation. Then, samples were homogenised for $10 \mathrm{~s}$ thrice using the Mini-Beadbeater (BioSpec Products, Bartlesville, OK, USA). After centrifugation (15 $\mathrm{min}, 4^{\circ} \mathrm{C}, 50,000 \times g$ ), the obtained supernatant was diluted 100-fold in water and placed in the high-performance liquid chromatography apparatus. Amino acids were measured after pre-column derivatisation using $o$-phthaldialdehyde (Thermo Fisher Scientific). The resulting derivatives were separated in the chromatography column using an acetonitrile gradient and detected using a fluorescence detector (Meesters et al., 2016; van Eijk et al., 1993).

\section{$\mu \mathrm{CT}$}

Before $\mu \mathrm{CT}$ measurements, plates and screws were carefully removed from the femur avoiding disruption of the newly formed callus.

Femora were scanned by $\mu \mathrm{CT}(\mu \mathrm{CT} 40$, Scanco Medical) at $70 \mathrm{kVp}$ and $114 \mu \mathrm{A}$ with a $200 \mathrm{~ms}$ integration time. A Gaussian filter (sigma 0.8, support 1) was used for a partial suppression of the noise. Based on the described grey values, the degree of mineralisation could be quantified. To ensure comparable measurements, bone volumes were measured between the first proximal and distal screw-holes next to the osteotomy. This region resembled 100 cross-sectional slices on the $\mu \mathrm{CT}$ image.

\section{Radiologic assessment}

Post-mortem cross-sectional $\mu \mathrm{CT}$ images of each femur were graded based on callus formation, rebridging of the cortex and remodelling of the callus by two blinded (bio)medically trained-investigators using the scoring scale previously described by Garrett et al. (2007) (Table 1).

\section{RNA analysis}

Crushed femur samples were incubated in TriSure (Bioline, London, UK) and homogenised using the Mini-Beadbeater, as mentioned before. Following homogenisation, RNA was precipitated using isopropanol and centrifugation $\left(30 \mathrm{~min}, 4{ }^{\circ} \mathrm{C}\right.$,

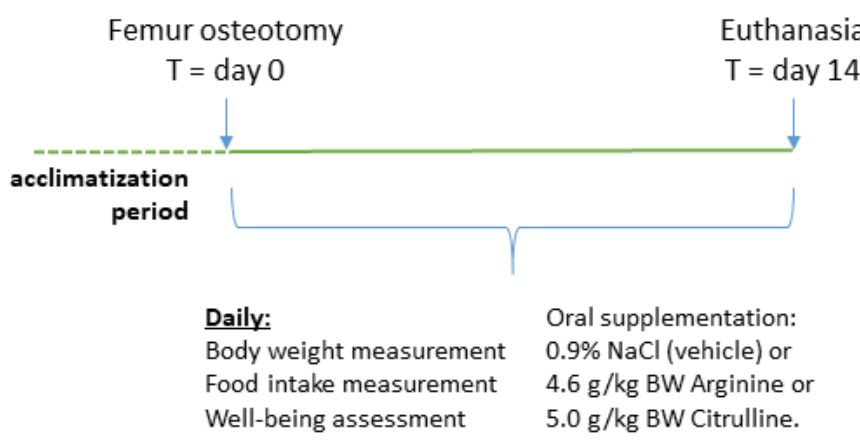

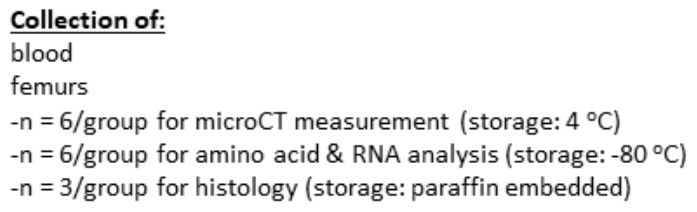

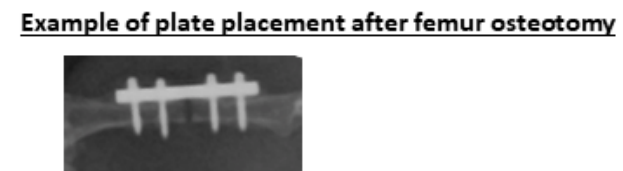

Fig. 2. Schematic representation of the experimental setup. After 2-week acclimatisation, a femur osteotomy was performed and, subsequently, mice were treated either as control or with arginine or citrulline supplementation daily for 2 weeks. Afterwards, mice were euthanised using cardiac puncture and samples were collected. 
Table 1. Radiographic scoring scale based on callus formation, rebridging of the cortices and remodelling of the callus.

\begin{tabular}{|c|c|}
\hline Score & Definition \\
\hline $\mathbf{0}$ & No bridging, no callus formation \\
\hline $\mathbf{1}$ & No bridging, initiation of small amount of callus \\
\hline $\mathbf{2}$ & No bridging, obvious initial callus formation near fracture \\
\hline $\mathbf{3}$ & Rebring, marked callus formation near and around fracture site \\
\hline $\mathbf{4}$ & Rebridging of at least one of the cortices, marked callus formation near and around fracture site \\
\hline $\mathbf{5}$ & Rebridging of at least one of the cortices, marked and complete callus formation around fracture site \\
\hline $\mathbf{6}$ & Clear rebridging of both cortices and resolution of the callus \\
\hline 7 & \\
\hline
\end{tabular}

Table 2. Primer sequences for qPCR. Fw: forward; Rev: reverse.

\begin{tabular}{|c|c|c|}
\hline Gene & Name & Primer sequence $\left(5^{\prime}-3^{\prime}\right)$ \\
\hline \multirow{2}{*}{ Ppia } & Cyclophilin A Fw & TTCСТССТTTCACAGAATTATTCCA \\
\hline & Cyclophilin A Rev & CCGCCAGTGCCATTATGG \\
\hline \multirow{2}{*}{ Actb } & $\beta$-actin Fw & GACAGGATGCAGAAGGAGATTACTG \\
\hline & $\beta$-actin Rev & CCACCGATCCACACAGAGTACTT \\
\hline \multirow{2}{*}{ Arg1 } & Arginase- $1 \mathrm{Fw}$ & GGAGAGCCTTCCTGCACTTT \\
\hline & Arginase-1 Rev & GTGCCTTGGTCTACATTGAACATAC \\
\hline \multirow{2}{*}{ Nos2 } & iNOS Fw & TTGCAAGCTGATGGTCAAGATC \\
\hline & iNOS Rev & CAACCCGAGCTCCTGGAA \\
\hline \multirow{2}{*}{ Nos3 } & eNOS Fw & TTAATGTGGCCGTGTTGCA \\
\hline & eNOS Rev & CTCTTGATGGAAGACAGGAGTTAGG \\
\hline \multirow{2}{*}{ Bmp2 } & BMP2 Fw & GCTTCTTAGACGGACTGCGG \\
\hline & BMP2 Rev & GCAACACTAGAAGACAGCGGGT \\
\hline \multirow{2}{*}{ Bmp7 } & BMP7 Fw & CCAAAGAACCAAGAGGCCC \\
\hline & BMP7 Rev & GCTGCTGTTTTCTGCCACACT \\
\hline \multirow{2}{*}{ Vegfa } & VEGF Fw & GCTTTACTGCTGTACCTCCACCA \\
\hline & VEGF Rev & GGGACTTCTGCTCTCCTTCTGTC \\
\hline \multirow{2}{*}{ Col2a1 } & Collagen $2 \mathrm{Fw}$ & GAGAGGTCTTCCTGGCAAAG \\
\hline & Collagen 2 Rev & AAGTCCCTGGAAGCCAGAT \\
\hline \multirow{2}{*}{ Il-6 } & Interleukin $6 \mathrm{Fw}$ & GCTACCAAACTGGATATAATCAGGAAA \\
\hline & Interleukin 6 Rev & CTTGTTATCTTTTAAGTTGTTCTTCATGTACTC \\
\hline \multirow{2}{*}{$N f k b 1$} & $\mathrm{NF}-\kappa \mathrm{B} F \mathrm{w}$ & GCTACGGCGGCCTTCTG \\
\hline & NF- $\kappa B$ Rev & CAATCCGGTGGCGATCAT \\
\hline \multirow{2}{*}{$I l-1 a$} & Interleukin $1 \alpha \mathrm{Fw}$ & AAAGAATCTATACCTGTCCTGTGTAATGAAA \\
\hline & Interleukin $1 \alpha \operatorname{Rev}$ & GGTATTGCTTGGGATCCACACT \\
\hline \multirow{2}{*}{ Cxcl2/Mip2 } & Mip2 Fw & GCGCTGTCAATGCCTGAAGA \\
\hline & Mip2 Rev & TTTGACCGCCCTTGAGAGTG \\
\hline
\end{tabular}

$12,850 \times g)$. Obtained RNA pellets were washed with $80 \%$ ethanol and air-dried before dissolution in diethylpyrocarbonate (DEPC)-treated water (Sigma-Aldrich). To obtain adequate results, genomic DNA was removed using a DNase I treatment kit (Promega). Afterwards, RNA was precipitated using $3 \mathrm{M}$ sodium acetate and RNA was dissolved in DEPC-treated water before cDNA synthesis was performed using the iScript cDNA synthesis kit (Biorad). To perform quantitative polymerase chain reaction (qPCR) analysis, diluted cDNA was added to a SYBR green mix with the appropriate forward and reverse primers (see Table 2 for primer sequences). Cyclophilin A (Ppia) and $\beta$-actin (Actb) were used as housekeeping genes. cDNA was amplified using the LC480 Lightcycler (Roche), following a 3-step program consisting of 40 cycles of denaturation $\left(95^{\circ} \mathrm{C}, 10 \mathrm{~s}\right)$, annealing $\left(60^{\circ} \mathrm{C}, 20 \mathrm{~s}\right)$ and elongation $\left(70{ }^{\circ} \mathrm{C}, 20 \mathrm{~s}\right)$. Expression of the different genes was analysed using the LC480 software. The geometric 
mean of the housekeeping gene expressions was used as a normalisation factor. Used primers were purchased from Sigma-Aldrich.

\section{Histological assessment of fracture repair}

After euthanasia, plates and screws were carefully removed from the femora and samples were fixed in $4 \%$ paraformaldehyde solution and decalcified using ethylenediaminetetraacetic acid (EDTA). Subsequently, femora were embedded in paraffin wax and $4 \mu \mathrm{m}$-thick sections were prepared. For staining, sections were first deparaffinised in xylene and rehydrated from graded ethanol (100, 96 and $70 \%$ ) to distilled water and, afterwards, stained using haematoxylin and eosin.

\section{Statistical analysis}

Statistical analysis of the obtained results was performed using GraphPad Prism 6. Normality of the results was checked using the Shapiro-Wilks test. All data are represented as means and standard error of the mean (SEM). ANOVA with post-hoc Bonferroni correction was used to assess statistical significance. A Kruskal-Wallis test was performed on the discontinuous healing score measurements. In all cases, $p$-values below 0.05 were considered statistically significant.

\section{Results}

\section{Food intake and weight}

Food intake during the $14 \mathrm{~d}$ period was comparable between the control group and both experimental groups ( $4.5 \mathrm{~g}$ of regular chow per mouse per day, no further data shown). However, significant differences in animal total body weight were observed (Fig. 3). During the initial post-operative days, animals in all

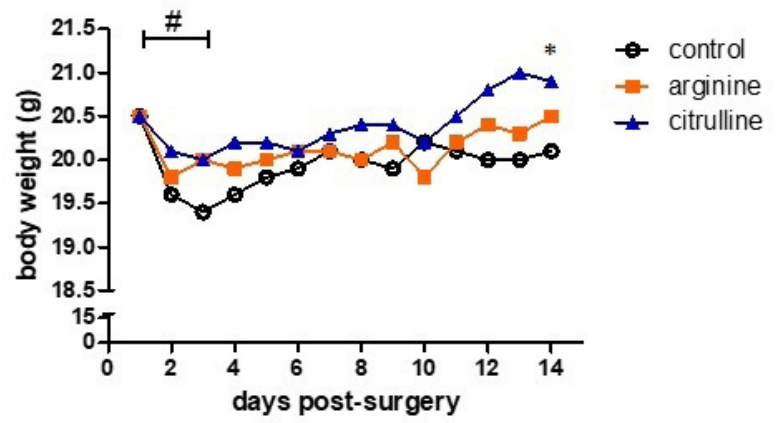

Fig. 3. Post-operative recovery. Average body weight per group per day. Mice in the control group are represented with the black line, argininetreated mice with orange and citrulline-treated mice with blue. SEM are not shown to increase readability of the Fig. \# marks a significant difference, $p<0.05$ in weight change within the first $2 \mathrm{~d}$ after the surgery between control mice and both treated groups. * marks a significant difference at day 14 between citrulline-treated mice and control animals. groups lost about $1 \mathrm{~g}$ of weight (5\% of total body weight). Over the complete $14 \mathrm{~d}$ experimental period, control animals lost $0.1 \mathrm{~g}$ of their initial body weight. Arginine-treated mice showed a body weight which was comparable with their pre-operative weight. Citrulline-treated mice showed an increase of $0.2 \mathrm{~g}$ over the $14 \mathrm{~d}$ period, which was significant when compared to control animals $(p<0.01)$. Additionally, both treated groups of mice showed a smaller decrease in body weight during the first 2 postoperative days when compared to control animals $(p<0.05)$.

\section{Callus formation}

Radiographic analysis showed that femora in all three groups healed with a relatively low variations in formed callus between the individual mice in each group. Fig. 4a shows representative threedimensional rendered images of femora after $\mu \mathrm{CT}$ analysis of the three experimental groups. According to the criteria mentioned in the Garrett scoring scale as presented in Table 1, after $14 \mathrm{~d}$ of healing, control mice showed a marked callus formation near the fracture site without bridging of the cortices. Almost all arginine-treated mice showed bridging of one of the cortices. In the citrulline-treated group all mice showed bridging of the cortices with beginning resolution of the callus, indicating a beginningremodelling phase. In addition, differences between citrulline-treated mice and the control group reached statistical significance ( $p<0.05$, Fig. 4 b). Fig. 4 c shows the bone volumes measured during the $\mu \mathrm{CT}$ analysis. These results agreed with the observed differences found using the radiographic scoring, based on the criteria defined in Table 1, with a statistical difference between samples obtained from the control and the citrulline-treated animals $(p=0.05)$. Fig. $4 \mathbf{d}$ shows representative microscopy images of histological slides after haematoxylin and eosin staining of femora of each experimental group. Findings regarding callus formation were in line with observations found by $\mu \mathrm{CT}$ analysis.

\section{Amino acid concentrations}

Arginine concentrations in femoral tissue of control animals were around $300 \mathrm{nmol} / \mathrm{g}$ of wet tissue. Treatment with arginine or citrulline did not result in significantly higher arginine concentrations in femur tissue in these groups (Fig. 5a). However, citrulline concentrations (Fig. 5b) increased significantly in mice that received $14 \mathrm{~d}$ citrulline supplementation when compared to control mice that only received the vehicle $(p<0.05)$. Between both treatment groups, a favourable trend in citrulline concentrations was observed following citrulline treatment $(p=0.053)$, compared to arginine treatment. The main influence of citrulline supplementation was observed on ornithine concentrations (Fig. 5c). In citrullinetreated animals, femur ornithine concentrations were significantly higher when compared to control $(p<0.05)$ and arginine-treated mice $(p<0.01)$. 


\section{qPCR}

Nos2 mRNA expression was significantly lower in femora of mice treated with arginine $(p<0.05)$ and citrulline $(p<0.0001)$ when compared with control mice. Additionally, citrulline supplementation seemed to downregulate Nos2 expression more than arginine supplementation $(p<0.001)$ (Fig. 6a). Although Nos3 expression showed a similar trend, no significant differences were found between the three groups (Fig. 6b). Also, for arginase-1 (Arg1) expression, no significant differences were found in the three groups ( $p=0.21$ between arginine- and citrulline-treated groups, Fig. 6c).

To assess the influence of bone morphogenetic proteins (BMPs), mRNA expression of Bmp2 and $B m p 7$ was measured. No significant differences were present in either of the groups for both genes (Fig. 6d,e).

Angiogenesis was assessed measuring the mRNA expression levels of the vascular endothelial growth factor A (Vegfa, Fig. 6f). Vegfa expression showed a significant 2-fold increase in citrulline-treated animals in comparison with control mice $(p<0.01)$. In addition, expression in citrulline-treated animals was also higher when compared with arginine-treated animals $(p<0.05)$.
Whereas Arg1 (a precursor for collagen synthesis) showed no differences between the groups, a difference was observed in collagen type II (Col2a1) expression. Col2a1 expression in citrulline-treated mice was higher when compared with both the control group and mice that received daily arginine supplementation (both $p<0.05$, Fig. 6g).

To further investigate the role of inflammation, interleukin-6 (Il-6), nuclear factor kappa B subunit 1 (Nfkb1), Il-1 and chemokine (C-X-C motif) ligand 2 (Cxcl2/Mip2) mRNA expression was measured (Fig. 6h,i,j,,k, respectively). Only Cxcl2/Mip2 showed significant results, with lower expression in arginine$(p<0.01)$ and citrulline-treated animals $(p<0.05)$ when compared to the control group.

\section{Discussion}

The present study showed that daily oral supplementation of citrulline in mice that underwent a controlled femur osteotomy increased new bone formation when compared with untreated mice. The arginine supplementation did not influence bone formation. Next to bone citrulline concentrations,

a

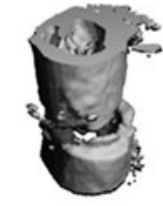

control



arginine

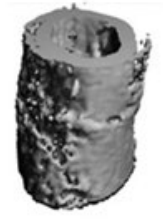

citrulline b

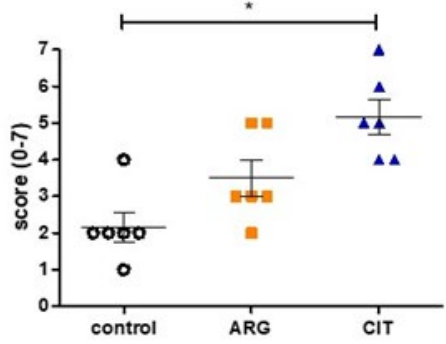

d

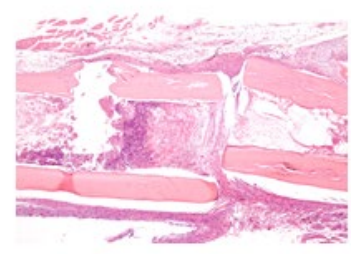

control



arginine c
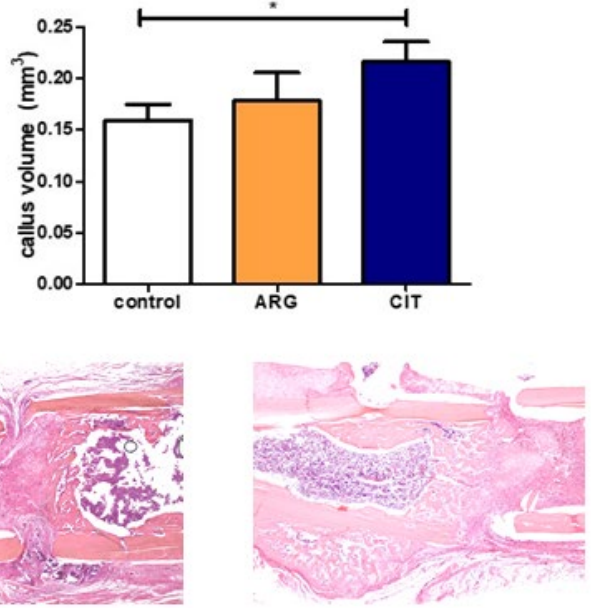

citrulline

Fig. 4. Callus formation. (a) Representative three-dimensional rendered images of callus formation in the three experimental groups from the osteotomy site towards the first proximal and distal screw-holes. (b) Quantification of callus formation using the Garrett scoring scale (0-7). (c) Quantification of bone volumes within the region represented in a. Legend: white represents the control group, orange arginine treatment, blue citrulline treatment. ${ }^{*} p<0.05$. (d) Representative images of histological haematoxylin and eosin staining of each treatment group. 
ornithine concentrations in the femora from citrullinetreated mice were also increased, possibly indicating the progress towards collagen synthesis through polyamine production. The increased ornithine concentrations were accompanied by an enhanced expression of arginase- 1 and a significant increase in collagen II expression. NOS enzymes showed lower expression profiles at the RNA level in citrullinetreated mice when compared with control mice. Increased expression of Vegfa provided insight into the significant improved angiogenesis after citrulline supplementation. Furthermore, mice treated with either of the amino acids showed an increased weight gain during the $14 \mathrm{~d}$ study period suggesting an improved post-operative recovery.

Generally, the absence of interfragmentary motion between two fracture parts in mice occurs after 3 weeks of bone healing (Garcia et al., 2013), suggesting an adequate callus formation and cortical bridging of the fracture gap. Subsequently, the process of callus resolution and remodelling will continue. The study hypothesis was that stimulation of the arginine-NO-citrulline metabolism would enhance fracture healing, thus shortening the period until the interfragmentary motion was absent. Therefore, a healing period of $14 \mathrm{~d}$ was decided upon to assess the outcome of metabolic stimulation in these mice.

Although several studies have already investigated the influence of a pharmacological (Diwan et al., 2000; Turner et al., 1996) or genetic (Meesters et al., 2016) disturbance in the arginine-citrulline-NO metabolism on the bone healing process, the present study reported for the first time the results of stimulation a

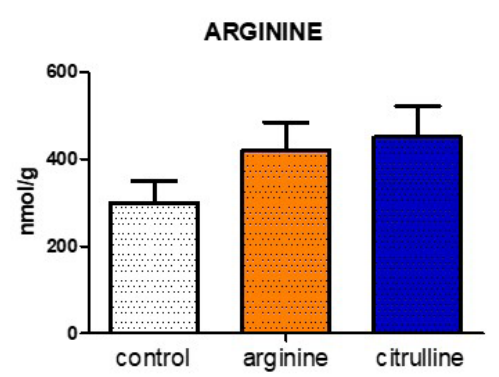

b

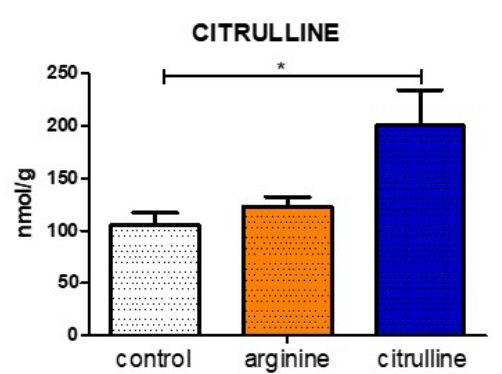

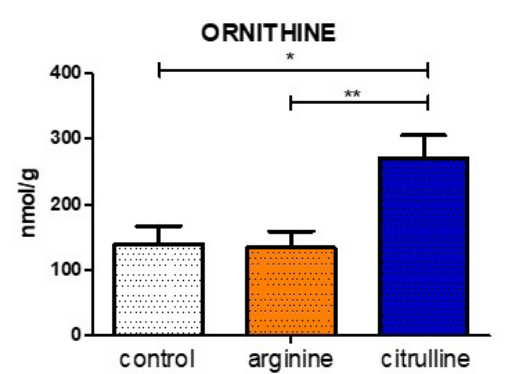

Fig. 5. Amino acid concentrations in femora. (a) Arginine, (b) citrulline and (c) ornithine concentrations in femoral callus tissue in $\mathrm{nmol} / \mathrm{g}$ measured after euthanasia of the animals. White bars represent control animals which received $0.9 \% \mathrm{NaCl}$ supplementation, orange bars arginine-treated mice, blue bars citrullinetreated mice. ${ }^{*} p<0.05,{ }^{* *} p<0.01$.

a

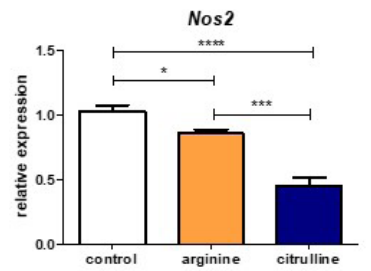

e

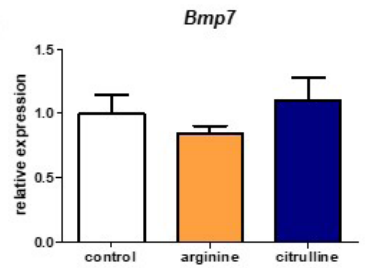

i

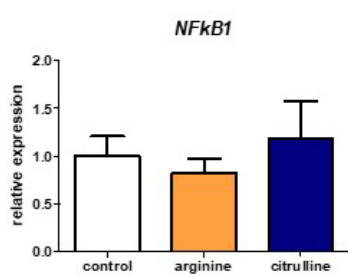

b

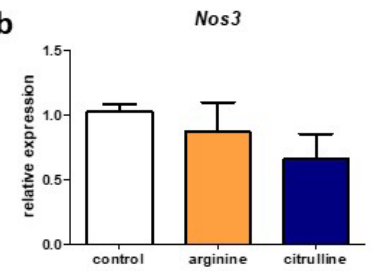

f

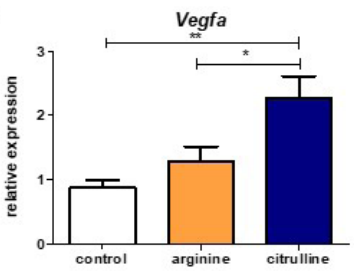

j

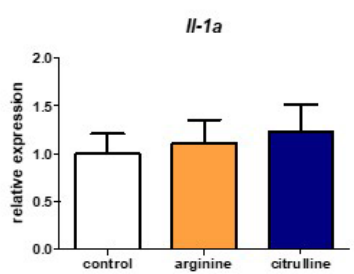

C

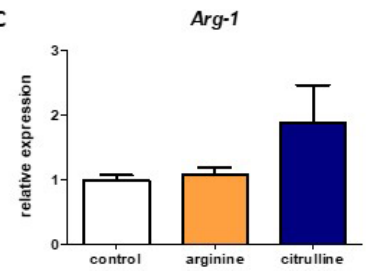

g
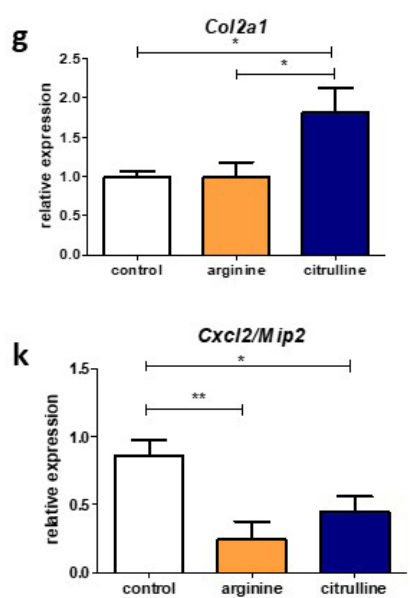


Fig. 6. mRNA expression in femoral tissue. (a) Nos2, (b) Nos3, (c) Arg1, (d) Bmp2, (e) Bmp7, (f) Vegfa, (g) Col2a1, (h) Il-6, (i) Nfkb1, (j) Il-1, (k) Cxcl2/Mip2. White bars represent controls, orange bars arginine-treated mice, blue bars citrulline-treated mice. ${ }^{*} p<0.05,{ }^{* *} p<0.01,{ }^{* * *} p<0.001,{ }^{* * * *} p<0.0001$. 
of bone healing in a reliable, representative and reproducible murine bone healing model.

In patients, availability of arginine and related amino acids is necessary to achieve adequate fracture healing (Wijnands et al., 2012a). Additionally, during prolonged hospitalisation after hip fracture surgery, elderly people mainly suffer a substantial loss of skeletal muscle mass (Kouw et al., 2019). Therefore, nutritional supplementation with amino acids is preferable perioperatively and during rehabilitation (Kramer et al., 2019). Additionally, mainly citrulline supplementation is known to increase skeletal muscle and total body weight in both in vivo animal experiments (Villareal et al., 2018) as well as in humans (Martone et al., 2015). The significant body weight gain and mainly the reduced weight loss during the first days, as observed in mice that were treated with citrulline, may point towards this enhanced post-operative recovery and was in line with previous animal research investigating the reversal of detrimental effects after protein malnutrition in bone healing (Day and DeHeer, 2001).

Amino acid measurements in femoral tissue showed both increased citrulline concentrations as well as ornithine concentrations after supplementation with citrulline when compared with tissues obtained from control mice. Conversion of arginine into ornithine is mediated by arginase- 1 . The results showed no significantly higher expression of arginase-1. However, through formation of polyamines, ornithine is a precursor for collagen synthesis, which was found to induce an increased mRNA expression in the femur samples. Under normal healing conditions, collagen II expression peaks after $14 \mathrm{~d}$ of bone healing (Grongroft et al., 2009) and gradually declines thereafter. One limitation of the study was the lack of multiple time points at which the influence of stimulation with either of the amino acids was measured. Therefore, future studies should focus on one or more time points ranging further in the healing process to assess the possible differences at later stages during the fracture healing process. Therefore, it is expected that collagen II expression would be higher when measured after $7 \mathrm{~d}$ of fracture healing in the present experimental setup.

Conversion of arginine into citrulline is mediated by the NOS enzymes. Mainly Nos2 and Nos3 are of influence during the initial healing responses. The neuronal Nos1 is only expressed during the later remodelling phase of fracture healing (Zhu et al., 2001), thus having no influence on the processes studied in the present study.

Nos2 expression was significantly lower in femora obtained from mice after citrulline treatment, indicating a beneficial and more advanced systemic inflammatory response after $14 \mathrm{~d}$ when compared with prolonged inflammation in the other experimental groups, which additionally was underlined by the results found in Cxcl2/Mip2 mRNA expression.
An adequate inflammatory response and fracture haematoma formation are necessary during the fracture healing process, with recruitment and activation of macrophages and, subsequently, neutrophils as well as production of pro-inflammatory cytokines, chemokines and growth factors. Disturbances to these inflammatory signalling processes often lead to development of delayed union and non-union later in the healing process (Bastian et al., 2016). Deletion of Nos2 results in prolonged inflammation and higher degrees of neutrophil influx in and around the fracture callus (Meesters et al., 2016) and, subsequently, in delayed union.

Nos3, mainly expressed in the endothelium during the vascular ingrowth of capillaries in and around the callus (Zhu et al., 2002), showed no significant differences between the studied groups. However, $V e g f a$ was significantly increased in citrullinetreated mice that showed increased bone formation, indicating an activation of angiogenesis while new vessels were not yet formed, explaining the no difference observed in Nos3 expression.

In the study model, a controlled mid shaft femur osteotomy was induced. However, patients who sustain a fracture usually show different fracture patters due to the mechanics related to the trauma. Another future perspective is to investigate stimulation of fracture healing by arginine-citrullineNO metabolism in a model more closely resembling these trauma mechanics and fracture patterns. Different small animal models with closed fracture induction methods are available (Histing et al., 2011; Holstein et al., 2009). Additionally, a translation into the human clinical setting is obviously the goal of the performed research. Although bone healing metabolism is comparable between mice and humans, small differences remain present next to differences in biomechanical loading, i.e. lack of a Haversian system in rodents and different kinetics in metabolic processes.

In conclusion, the study showed that daily oral citrulline supplementation beneficially affected the bone healing response after a controlled femur osteotomy in mice.

\section{Acknowledgements}

The authors gratefully acknowledge Dr Bert van Rietbergen (Technical University Eindhoven) for his assistance during the $\mu \mathrm{CT}$ analysis and Dr Stephan Zeiter (AO Research Institute Davos) for sharing his knowledge on the surgical procedures that were performed in the study.

All authors declare that they do not have any conflicts of interest regarding the research presented in the manuscript. 


\section{References}

Aaron RK, Ciombor DM, Simon BJ (2004) Treatment of nonunions with electric and electromagnetic fields. Clin Orthop Relat Res 419: 21-29.

Bastian OW, Kuijer A, Koenderman L, Stellato RK, van Solinge WW, Leenen LP, Blokhuis TJ (2016) Impaired bone healing in multitrauma patients is associated with altered leukocyte kinetics after major trauma. J Inflamm Res 9: 69-78.

Bishop JA, Palanca AA, Bellino MJ, Lowenberg DW (2012) Assessment of compromised fracture healing. J Am Acad Orthop Surg 20: 273-282.

Calori GM, Albisetti W, Agus A, Iori S, Tagliabue L (2007) Risk factors contributing to fracture nonunions. Injury 38 Suppl 2: S11-18.

Chae HJ, Park RK, Chung HT, Kang JS, Kim MS, Choi DY, Bang BG, Kim HR (1997) Nitric oxide is a regulator of bone remodelling. J Pharm Pharmacol 49: 897-902.

Corbett SA, McCarthy ID, Batten J, Hukkanen M, Polak JM, Hughes SP (1999) Nitric oxide mediated vasoreactivity during fracture repair. Clin Orthop Relat Res 365: 247-253.

Court-Brown CM, McQueen MM (2008) Nonunions of the proximal humerus: their prevalence and functional outcome. J Trauma 64: 1517-1521.

Day SM, DeHeer DH (2001) Reversal of the detrimental effects of chronic protein malnutrition on long bone fracture healing. J Orthop Trauma 15: 47-53.

Diwan AD, Wang MX, Jang D, Zhu W, Murrell GA (2000) Nitric oxide modulates fracture healing. J Bone Miner Res 15: 342-351.

Garcia P, Histing T, Holstein JH, Klein M, Laschke MW, Matthys R, Ignatius A, Wildemann B, Lienau J, Peters A, Willie B, Duda G, Claes L, Pohlemann T, Menger MD (2013) Rodent animal models of delayed bone healing and non-union formation: a comprehensive review. Eur Cell Mater 26: 1-14.

Garrett IR, Gutierrez GE, Rossini G, Nyman J, McCluskey B, Flores A, Mundy GR (2007) Locally delivered lovastatin nanoparticles enhance fracture healing in rats. J Orthop Res 25: 1351-1357.

Grongroft I, Heil P, Matthys R, Lezuo P, Tami A, Perren S, Montavon P, Ito K (2009) Fixation compliance in a mouse osteotomy model induces two different processes of bone healing but does not lead to delayed union. J Biomech 42: 2089-2096.

Grongroft I, Wissing S, Meesters DM, Poeze M, Matthys-Mark R, Ito K, Zeiter S (2019) Development of a novel murine delayed secondary fracture healing in vivo model using periosteal cauterization. Arch Orthop Trauma Surg 139: 1743-1753.

Histing T, Garcia P, Holstein JH, Klein M, Matthys R, Nuetzi R, Steck R, Laschke MW, Wehner T, Bindl R, Recknagel S, Stuermer EK, Vollmar B, Wildemann B, Lienau J, Willie B, Peters A, Ignatius A, Pohlemann T, Claes L, Menger MD (2011) Small animal bone healing models: standards, tips, and pitfalls results of a consensus meeting. Bone 49: 591-599.

Holstein JH, Matthys R, Histing T, Becker SC, Fiedler M, Garcia P, Meier C, Pohlemann T, Menger MD (2009) Development of a stable closed femoral fracture model in mice. J Surg Res 153: 71-75.

Kouw IWK, Groen BBL, Smeets JSJ, Kramer IF, van Kranenburg JMX, Nilwik R, Geurts JAP, Ten Broeke RHM, Poeze M, van Loon LJC, Verdijk LB (2019) One week of hospitalization following elective hip surgery induces substantial muscle atrophy in older patients. J Am Med Dir Assoc 20: 35-42.

Kramer IF, Blokhuis TJ, Verdijk LB, van Loon LJC, Poeze M (2019) Perioperative nutritional supplementation and skeletal muscle mass in older hip-fracture patients. Nutr Rev 77: 254-266.

Martone AM, Lattanzio F, Abbatecola AM, Carpia DL, Tosato M, Marzetti E, Calvani R, Onder G, Landi F (2015) Treating sarcopenia in older and oldest old. Curr Pharm Des 21: 1715-1722.

Meesters DM, Neubert S, Wijnands KA, Heyer FL, Zeiter S, Ito K, Brink PR, Poeze M (2016) Deficiency of inducible and endothelial nitric oxide synthase results in diminished bone formation and delayed union and non-union development. Bone 83: 111-118.

Meesters DM, Wijnands KAP, Brink PRG, Poeze M (2018) Malnutrition and fracture healing: are specific deficiencies in amino acids important in nonunion development? Nutrients 10. pii: E1597. DOI: 10.3390/ nu10111597.

Mills LA, Aitken SA, Simpson A (2017) The risk of non-union per fracture: current myths and revised figures from a population of over 4 million adults. Acta Orthop 88: 434-439.

Poeze M, Bruins MJ, Kessels F, Luiking YC, Lamers WH, Deutz NE (2011) Effects of L-arginine pretreatment on nitric oxide metabolism and hepatosplanchnic perfusion during porcine endotoxemia. Am J Clin Nutr 93: 1237-1247.

Turner CH, Takano Y, Owan I, Murrell GA (1996) Nitric oxide inhibitor L-NAME suppresses mechanically induced bone formation in rats. Am J Physiol 270: E634-639.

van Eijk HM, Rooyakkers DR, Deutz NE (1993) Rapid routine determination of amino acids in plasma by high-performance liquid chromatography with a 2-3 microns Spherisorb ODS II column. J Chromatogr 620: 143-148.

van Staa TP, Dennison EM, Leufkens HG, Cooper C (2001) Epidemiology of fractures in England and Wales. Bone 29: 517-522.

Villareal MO, Matsukawa T, Isoda H (2018) L-citrulline supplementation-increased skeletal muscle pgc-1alpha expression is associated with exercise performance and increased skeletal muscle weight. Mol Nutr Food Res: e1701043. DOI: 10.1002/ mnfr.201701043.

Vittur F, Lunazzi G, Moro L, Stagni N, de Bernard B, Moretti M, Stanta G, Bacciottini F, Orlandini G, Reali N, Amos Casti (1986) A possible role 
for polyamines in cartilage in the mechanism of calcification. Biochim Biophys Acta 881: 38-45.

Wijnands KA, Brink PR, Weijers PH, Dejong CH, Poeze M (2012a) Impaired fracture healing associated with amino acid disturbances. Am J Clin Nutr 95: 1270-1277.

Wijnands KA, Vink H, Briede JJ, van Faassen EE, Lamers WH, Buurman WA, Poeze M (2012b) Citrulline a more suitable substrate than arginine to restore no production and the microcirculation during endotoxemia. PLoS One 7: e37439. DOI: 10.1371/journal.pone.0037439.

Zhu W, Diwan AD, Lin JH, Murrell GA (2001) Nitric oxide synthase isoforms during fracture healing. J Bone Miner Res 16: 535-540.

Zhu W, Murrell GA, Lin J, Gardiner EM, Diwan AD (2002) Localization of nitric oxide synthases during fracture healing. J Bone Miner Res 17: 14701477.

\section{Discussion with Reviewer}

Simon Chow: Based on the study results, what is the authors' hypothesis on the effect of citrulline supplementation on the acute inflammatory response at the fracture site at a shorter time-point?
Authors: Where qPCR measurements of the inflammatory markers did not all show a significant difference after citrulline supplementation when measured after $14 \mathrm{~d}$, we hypothesise the differences to be more pronounced at a shorter time-point, since the inflammatory response usually peaks during the first days of the fracture healing process.

Simon Chow: What is the authors' recommendation in relation to the current findings about fragilityfracture patients generally found to be in sub-optimal nutritional states?

Authors: The obvious first choice would be to perform a randomised trial with such patients investigating the influence of citrulline supplementation extrapolated from the present animal study - and comparing it with a control group. Additionally, the influence of citrulline supplementation in patients which are at risk for fragility-fractures should be investigated to determine if the fracture risk would already decrease due to beneficial influences on muscle metabolism and function.

Editor's note: The Scientific Editor responsible for this paper was Chris Evans. 